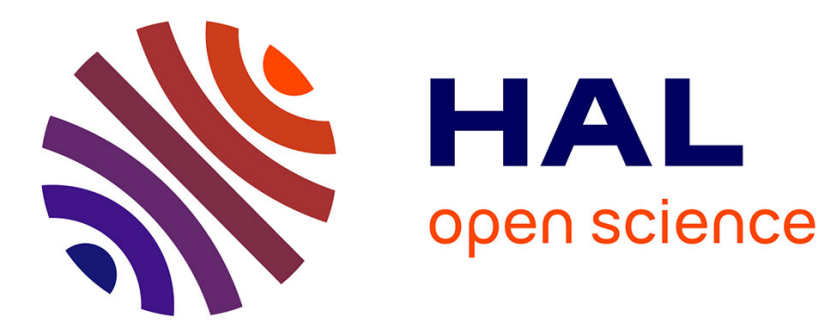

\title{
Dynamic modeling and control of a tensegrity manipulator mimicking a bird neck
}

Benjamin Fasquelle, Matthieu Furet, Christine Chevallereau, Philippe Wenger

\section{To cite this version:}

Benjamin Fasquelle, Matthieu Furet, Christine Chevallereau, Philippe Wenger. Dynamic modeling and control of a tensegrity manipulator mimicking a bird neck. Advances in Mechanism and Machine ScienceProceedings of the 15th IFToMM World Congress on Mechanism and Machine Science, pp.20872097, 2019, 978-3-030-20130-2. 10.1007/978-3-030-20131-9_207. hal-02353763

\section{HAL Id: hal-02353763 https://hal.science/hal-02353763}

Submitted on 13 Nov 2019

HAL is a multi-disciplinary open access archive for the deposit and dissemination of scientific research documents, whether they are published or not. The documents may come from teaching and research institutions in France or abroad, or from public or private research centers.
L'archive ouverte pluridisciplinaire HAL, est destinée au dépôt et à la diffusion de documents scientifiques de niveau recherche, publiés ou non, émanant des établissements d'enseignement et de recherche français ou étrangers, des laboratoires publics ou privés. 


\title{
Dynamic modeling and control of a tensegrity manipulator mimicking a bird neck
}

\author{
Benjamin Fasquelle, Matthieu Furet, Christine Chevallereau, and Philippe \\ Wenger \\ Laboratoire des Sciences du Numérique de Nantes (LS2N), CNRS, Ecole centrale de \\ Nantes, 44321 Nantes, France
}

\begin{abstract}
This paper studies a tensegrity manipulator mimicking a bird neck. This manipulator is built upon assembling several X-shape one-dof tensegrity mechanisms in series. A methodology is proposed to derive the dynamic model using Lagrange's equations. The dynamic model is used to design a dynamic control law. This control law is applied to a backward-and-forward motion between an S-shape rest equilibrium configuration and a straight configuration of the neck manipulator. Simulation results show a much better tracking as compared with a classical PD control.
\end{abstract}

Keywords: Tensegrity, bird neck model, dynamic control

\section{Introduction}

The bird neck features outstanding dexterity and dynamic performances (think of the woodpecker). It operates in large workspaces and applies high forces and torques relative to the bird mass. Contrary to hydrostats such as the elephant trunk or the cephalopod tentacle, bird necks have a spine like the snake, but they do not lie on the ground like those latter. This work is carried out in the frame of a collaborative, multidisciplinary project and aims at proposing a bird neck robotic model. The concept of tensegrity has been chosen in this project as a general paradigm able to link the interests of biologists and roboticists. A tensegrity structure is made of compressive and tensile components held together in equilibrium [1], [2]. Tensegrity structures were first used in art [3] and have then been applied in civil engineering [4] and robotics [5], [6], [7], [8]. There are suitable to model muskuloskeleton structures where the bones are the compressive components and the muscles and tendons are the tensile elements [9]. A preliminary, planar bird neck robotic model is considered in this paper. This model is built upon stacking a series of Snelson's X-shape mechanisms [2]. Although simplified because it is planar, this model goes beyond the only available bird neck model in the literature that uses a simple planar articulated linkage [10]. Snelson's X-shape mechanisms have been studied by a number of researchers, either as a single mechanism [5], [7], [11], [12] or assembled in series [13], [14], [15], [16], [17], [18]. In this paper, each X-shape mechanism is actuated with two 
lateral tendons threaded through the spring attachment points like in [15], [16], [17], and [18]. The resulting manipulator is supposed to operate in a vertical plane and is thus subject to gravity, unlike in [15], where the mechanism was used in a snake-like manipulator moving on the ground. The goal of this study is to propose a dynamic model of the manipulator along with a dynamic control law. The control law is simulated for point-to-point motions between two desired configurations.

\section{A tensegrity-based neck model}

We consider a set of $N$ X-shape tensegrity mechanisms stacked in series (see figure 1). The mechanisms $i$ are numbered from $i=1$ (base) to $i=N$ (head). Each mechanism $i$ is a class-2 tensegrity mechanism [4] consisting of a bottom bar $\mathcal{B}_{1 i}$, a top bar $\mathcal{B}_{4 i}$, two crossed bars $\left(\mathcal{B}_{2 i}\right.$ and $\left.\mathcal{B}_{3 i}\right)$ and two pretensioned springs. Note that the bottom bar of mechanism $i$ and the top bar of mechanism $i-1$ are the same, namely, $\mathcal{B}_{1 i}=\mathcal{B}_{4(i-1)}$. The first mechanism is fixed to the ground and all the rigid links and springs are connected to each other with perfect revolute joints. All the crossed bars have the same length $L$ and all the top and bottom bars have the same length $b$, which means that the mechanism is an anti-parallelogram. In parallel to the left spring (resp. to the right spring), a tendon (not shown in figure 1) applies a force $f_{l_{i}}\left(\right.$ resp. $f_{r_{i}}$ ).

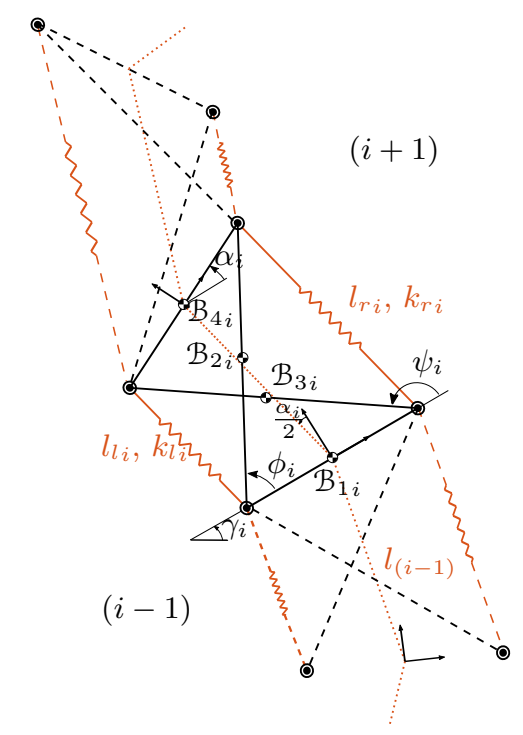

Fig. 1: Parametrization of a stack of mechanisms.

The angle parameters are shown in figure 1. $\boldsymbol{\alpha}=\left[\alpha_{1}, \ldots, \alpha_{N}\right]^{\top}$ is chosen as the vector of generalized coordinates, where $\alpha_{i}$ is the angle between the bottom bar 
$\mathcal{B}_{1 i}$ and the top bar $\mathcal{B}_{4 i}$. The other angles $\phi_{i}$ and $\psi_{i}$ are useful for computation and can be expressed w.r.t $\alpha_{i}$ (equation (1)) [12].

$$
\begin{aligned}
& \phi_{i}\left(\alpha_{i}\right)=2 \arctan \left[\frac{2 b L \sin \left(\alpha_{i}\right)+S}{\left(2 b^{2}+2 b L\right)\left[\cos \left(\alpha_{i}\right)+1\right]}\right] \\
& \psi_{i}\left(\alpha_{i}\right)=2 \arctan \left[\frac{-2 b L \sin \left(\alpha_{i}\right)-S}{\left(2 b^{2}-2 b L\right)\left[\cos \left(\alpha_{i}\right)+1\right]}\right]
\end{aligned}
$$

with $S=\sqrt{\left(-2 b L \sin \left(\alpha_{i}\right)\right)^{2}+\left(-2 b L\left[\cos \left(\alpha_{i}\right)+1\right]\right)^{2}-\left(2 b^{2}\left[\cos \left(\alpha_{i}\right)+1\right]\right)^{2}}$.

In order to have a generic formula, let $\gamma_{i}$ denote the angle of the base bar of the $i^{\text {th }}$ mechanism with respect to the horizontal axis:

$$
\gamma_{i+1}=\gamma_{i}+\alpha_{i}, \quad i=1, \ldots, N
$$

Note that $\gamma_{1}$, the orientation of the first mechanism, is given as a geometric parameter of the neck and depends on the bird specie.

\section{Dynamic modelling}

The Lagrange's equations for a multi-dof system are:

$$
\frac{d}{d t}\left(\frac{\partial T}{\partial \dot{\alpha}_{i}}\right)-\frac{\partial T}{\partial \alpha_{i}}+\frac{d V}{d \alpha_{i}}+=Q_{i}, \quad i=1,2, \ldots, n_{\mathrm{dof}}
$$

where $\mathrm{T}$ is the kinetic energy, $\mathrm{V}$ the potential energy, $\mathrm{Q}$ the generalized forces and $n_{\text {dof }}$ is the number of dof. In the next sections the kinetic and potential energy and the generalized forces used in (3) are derived to obtain the equation of motion in the following form:

$$
\mathbf{M}(\boldsymbol{\alpha}) \ddot{\boldsymbol{\alpha}}+\mathbf{C}(\boldsymbol{\alpha}, \dot{\boldsymbol{\alpha}}) \dot{\boldsymbol{\alpha}}+\mathbf{G}(\boldsymbol{\alpha})=\mathbf{Q}(\boldsymbol{\alpha})
$$

where $\mathbf{M}$ is the inertia matrix, such that $T=\frac{1}{2} \dot{\boldsymbol{\alpha}}^{\top} \mathbf{M} \dot{\boldsymbol{\alpha}}, \mathbf{C}$ is the matrix of coriolis effects that can be deduced from $\mathbf{M}$ [19], $\mathbf{G}$ is the vector of potential effects, $\mathbf{G}=\frac{d V}{d \boldsymbol{\alpha}}$, and $\mathbf{Q}$ is the vector of generalized forces.

\subsection{Kinetic energy and inertia Matrix}

The inertia matrix for one mechanism is first computed. The local frame of mechanism $i$ is located at the center $x_{i}, y_{i}$ of the base bar with the $\mathrm{x}$-axis along this bar, forming an angle $\gamma_{i}$ with the horizontal axis. Thus, the local frame of each mechanism is described by the three parameters $x_{i}, y_{i}, \gamma_{i}$. The translational (resp. rotational) velocities are $\dot{x}_{i}, \dot{y}_{i}\left(\right.$ resp. $\left.\dot{\gamma}_{i}\right)$. Since the position and orientation do not affect the kinetic energy, $x_{i}, y_{i}, \gamma_{i}$ will not appear in the equation. The total kinetic energy of mechanism $i$ is obtained by summation of the translational and rotational kinetic energy of the three movable links $\mathcal{B}_{2 i}, \mathcal{B}_{3 i}$ and $\mathcal{B}_{4 i}$ : 


$$
T_{i}=\frac{1}{2}\left(m_{2 i} v_{2 i}{ }^{2}+I_{2 i}\left(\dot{\phi}_{i}+\dot{\gamma}_{i}\right)^{2}+m_{3 i} v_{3 i}{ }^{2}+I_{3 i}\left(\dot{\psi}_{i}+\dot{\gamma}_{i}\right)^{2}+m_{4 i} v_{4 i}^{2}+I_{4 i}\left(\dot{\alpha}_{i}+\dot{\gamma}_{i}\right)^{2}\right)
$$

where $m_{j i}$ and $I_{j i}$ are the mass and inertia of bar $\mathcal{B}_{j i}$ and $v_{j i}$ is the velocity of body $\mathcal{B}_{j i}$ CoM. The first (resp. second) term in every set of brackets corresponds to the translational (resp. rotational) kinetic energy. Note that by definition $x_{i+1}=x_{4 i}$ and $y_{i+1}=y_{4 i}$. Let us define $l_{i}^{2}=\left(x_{4 i}-x_{i}\right)^{2}+\left(y_{4 i}-y_{i}\right)^{2}$. It can be shown that $l_{i}$ does not depend on $\gamma_{i}$ but it is a function of $\alpha_{i}$ [17]: $l_{i}\left(\alpha_{i}\right)=\sqrt{L^{2}-b^{2} \cos \left(\frac{\alpha_{i}}{2}\right)^{2}}$. The absolute coordinates of the pose of the centre of mass of segments $2,3,4$ are:

$$
\begin{aligned}
x_{2 i} & =x_{i}-\frac{b}{2} \cos \left(\gamma_{i}\right)+\frac{L}{2} \cos \left(\gamma_{i}+\phi_{i}\right) \\
y_{2 i} & =y_{i}-\frac{b}{2} \sin \left(\gamma_{i}\right)+\frac{L}{2} \sin \left(\gamma_{i}+\phi_{i}\right) \\
x_{3 i} & =x_{i}+\frac{b}{2} \cos \left(\gamma_{i}\right)+\frac{L}{2} \cos \left(\gamma_{i}+\psi_{i}\right) \\
y_{3 i} & =y_{i}+\frac{b}{2} \sin \left(\gamma_{i}\right)+\frac{L}{2} \sin \left(\gamma_{i}+\psi_{i}\right) \\
x_{4 i} & =x_{i+1}=x_{i}-l_{i}\left(\alpha_{i}\right) \sin \left(\gamma_{i}+\frac{\alpha_{i}}{2}\right) \\
y_{4 i} & =y_{i+1}=y_{i}+l_{i}\left(\alpha_{i}\right) \cos \left(\gamma_{i}+\frac{\alpha_{i}}{2}\right)
\end{aligned}
$$

By differentiating (6) and using (5), the total kinetic energy of mechanism $i$ can be written in the following form :

$$
T_{i}=\frac{1}{2}\left[\dot{x}_{i} \dot{y}_{i} \dot{\gamma}_{i} \dot{\alpha}_{i}\right] \mathbf{M}_{i}\left[\begin{array}{c}
\dot{x}_{i} \\
\dot{y}_{i} \\
\dot{\gamma}_{i} \\
\dot{\alpha}_{i}
\end{array}\right]
$$

with $\mathbf{M}_{i}$ such that:

$$
\mathbf{M}_{i}=\left[\begin{array}{cccc}
M_{x x i} & 0 & M_{\gamma x i} & M_{\alpha x i} \\
0 & M_{y y i} & M_{\gamma y i} & M_{\alpha y i} \\
M_{\gamma x i} & M_{\gamma y i} & M_{\gamma \gamma i} & M_{\alpha \gamma i} \\
M_{\alpha x i} & M_{\alpha y i} & M_{\alpha \gamma i} & M_{\alpha \alpha i}
\end{array}\right]
$$

where:

$$
\begin{aligned}
M_{\alpha \alpha i}= & m_{2 i}\left(\frac{L^{2} S_{2 i}^{2}\left(\alpha_{i}\right)}{4}\right)+m_{3 i}\left(\frac{L^{2} S_{3 i}^{2}\left(\alpha_{i}\right)}{4}\right)+m_{4 i}\left(\left(\frac{d l_{i}\left(\alpha_{i}\right)}{d \alpha_{i}}\right)^{2}+\frac{l_{i}^{2}\left(\alpha_{i}\right)}{4}\right)+ \\
& S_{2 i}^{2}\left(\alpha_{i}\right) I_{2 i}+S_{3 i}^{2}\left(\alpha_{i}\right) I_{3 i}+I_{4 i} \\
M_{\gamma \gamma i}= & m_{2 i}\left(\frac{b^{2}}{4}+\frac{L^{2}}{4}-\frac{b L}{2} \cos \left(\phi_{i}\right)\right)+m_{3 i}\left(\frac{b^{2}}{4}+\frac{L^{2}}{4}-\frac{b L}{2} \cos \left(\psi_{i}\right)\right)+m_{4 i}\left(l_{i}^{2}\left(\alpha_{i}\right)\right)+ \\
& I_{2 i}+I_{3 i}+I_{4 i} \\
M_{\alpha \gamma i}= & m_{2 i}\left(\frac{L^{2}}{4} S_{2 i}\left(\alpha_{i}\right)-\frac{b L}{4} S_{2 i}\left(\alpha_{i}\right) \cos \left(\phi_{i}\right)\right)+m_{3 i}\left(\frac{L^{2}}{4} S_{3 i}\left(\alpha_{i}\right)-\frac{b L}{4} S_{3 i}\left(\alpha_{i}\right) \cos \left(\psi_{i}\right)\right)+ \\
& m_{4 i}\left(\frac{l_{i}^{2}\left(\alpha_{i}\right)}{4}\right)+S_{2 i}\left(\alpha_{i}\right) I_{2 i}+S_{3 i}\left(\alpha_{i}\right) I_{3 i}+I_{4 i}
\end{aligned}
$$




$$
\begin{aligned}
M_{\alpha x i}= & -m_{2 i}\left(\frac{L}{2} S_{2 i}\left(\alpha_{i}\right) \sin \left(\gamma_{i}+\phi_{i}\right)\right)-m_{3 i}\left(\frac{L}{2} S_{3 i}\left(\alpha_{i}\right) \sin \left(\gamma_{i}+\psi_{i}\right)\right)+ \\
& m_{4 i}\left(\frac{d l_{i}\left(\alpha_{i}\right)}{d \alpha_{i}} \sin \left(\gamma_{i}+\frac{\alpha_{i}}{2}\right)-\frac{l_{i}\left(\alpha_{i}\right)}{2} \cos \left(\gamma_{i}+\frac{\alpha_{i}}{2}\right)\right) \\
M_{\alpha y i}= & m_{2 i}\left(\frac{L}{2} S_{2 i}\left(\alpha_{i}\right) \cos \left(\gamma_{i}+\phi_{i}\right)\right)+m_{3 i}\left(\frac{L}{2} S_{3 i}\left(\alpha_{i}\right) \cos \left(\gamma_{i}+\psi_{i}\right)\right)+ \\
& m_{4 i}\left(\frac{d l_{i}\left(\alpha_{i}\right)}{d \alpha_{i}} \cos \left(\gamma_{i}+\frac{\alpha_{i}}{2}\right)-\frac{l_{i}\left(\alpha_{i}\right)}{2} \sin \left(\gamma_{i}+\frac{\alpha_{i}}{2}\right)\right) \\
M_{\gamma x i}= & m_{2 i}\left(\frac{b}{2} \sin \left(\gamma_{i}\right)-\frac{L}{2} \sin \left(\gamma_{i}+\phi_{i}\right)\right)+m_{3 i}\left(\frac{b}{2} \sin \left(\gamma_{i}\right)-\frac{L}{2} \sin \left(\gamma_{i}+\psi_{i}\right)\right)+ \\
& m_{4 i}\left(-l_{i}\left(\alpha_{i}\right) \cos \left(\gamma_{i}+\frac{\alpha_{i}}{2}\right)\right) \\
M_{\gamma y i}= & m_{2 i}\left(-\frac{b}{2} \cos \left(\gamma_{i}\right)+\frac{L}{2} \cos \left(\gamma_{i}+\phi_{i}\right)\right)+m_{3 i}\left(-\frac{b}{2} \cos \left(\gamma_{i}\right)+\frac{L}{2} \cos \left(\gamma_{i}+\psi_{i}\right)\right)+ \\
& m_{4 i}\left(-l_{i}\left(\alpha_{i}\right) \sin \left(\gamma_{i}+\frac{\alpha_{i}}{2}\right)\right) \\
M_{x x i}= & M_{y y i}=m_{2 i}+m_{3 i}+m_{4 i}
\end{aligned}
$$

with $S_{2 i}\left(\alpha_{i}\right)=\frac{d \phi_{i}}{d \alpha_{i}}$ and $S_{3 i}\left(\alpha_{i}\right)=\frac{d \psi_{i}}{d \alpha_{i}}$.

Let us define a general transformation matrix $\mathbf{R}_{i}$ linking $\left[\dot{x}_{i}, \dot{y}_{i}, \dot{\gamma}_{i}, \dot{\alpha}_{i}\right]$ to the generalized coordinates $\left[\dot{\alpha}_{1}, \ldots \dot{\alpha}_{N}\right]$. this transformation matrix will be useful to build the inertia matrix of the whole manipulator:

$$
\left[\dot{x}_{i}, \dot{y}_{i}, \dot{\gamma}_{i}, \dot{\alpha}_{i}\right]^{\top}=\mathbf{R}_{i}\left[\dot{\alpha}_{1}, \ldots, \dot{\alpha}_{N}\right]^{\top}
$$

The translational and rotational velocities are calculated upon differenting eqs. (2), (6e) and (6f) with respect to time:

$$
\begin{aligned}
& \dot{x}_{i+1}=\dot{x}_{i}-l_{i} \cos \left(\gamma_{i}+\frac{\alpha_{i}}{2}\right) \dot{\gamma}_{i}+\left(-\frac{d l_{i}}{d \alpha_{i}} \sin \left(\gamma_{i}+\frac{\alpha_{i}}{2}\right)-\frac{l_{i}}{2} \cos \left(\gamma_{i}+\frac{\alpha_{i}}{2}\right)\right) \dot{\alpha}_{i} \\
& \dot{y}_{i+1}=\dot{y}_{i}-l_{i} \sin \left(\gamma_{i}+\frac{\alpha_{i}}{2}\right) \dot{\gamma}_{i}+\left(\frac{d l_{i}}{d \alpha_{i}} \cos \left(\gamma_{i}+\frac{\alpha_{i}}{2}\right)-\frac{l_{i}}{2} \sin \left(\gamma_{i}+\frac{\alpha_{i}}{2}\right)\right) \dot{\alpha}_{i} \\
& \dot{\gamma}_{i+1}=\dot{\gamma}_{i}+\dot{\alpha}_{i}
\end{aligned}
$$

Since $\dot{x}_{1}=\dot{y}_{1}=\dot{\gamma}_{1}=0$, the first transformation matrix $\mathbf{R}_{1}$ is:

$$
\mathbf{R}_{1}=\left[\begin{array}{c|c}
0 & \\
0 & \mathbf{0}_{4 \times(N-1)} \\
0 & \\
1 &
\end{array}\right]
$$

Matrix $\mathbf{R}_{i}$ can be defined by an iterative procedure. Using (2), equation (10) is rewritten under the form below:

$$
\left[\begin{array}{c}
\dot{x}_{i+1} \\
\dot{y}_{i+1} \\
\dot{\gamma}_{i+1} \\
\dot{\alpha}_{i+1}
\end{array}\right]=\left[\begin{array}{c}
\dot{x}_{i} \\
\dot{y}_{i} \\
\dot{\gamma}_{i} \\
\dot{\alpha}_{i}
\end{array}\right]+\left[\begin{array}{c}
x_{\gamma i} \\
y_{\gamma i} \\
0 \\
0
\end{array}\right]\left(\dot{\alpha}_{1}+\ldots+\dot{\alpha}_{i-1}\right)+\left[\begin{array}{c}
x_{\alpha i} \\
y_{\alpha i} \\
1 \\
-1
\end{array}\right] \dot{\alpha}_{i}+\left[\begin{array}{c}
0 \\
0 \\
0 \\
1
\end{array}\right] \dot{\alpha}_{i+1}
$$


with $x_{\gamma i}=-l_{i} \cos \left(\gamma_{i}+\frac{\alpha_{i}}{2}\right), x_{\alpha i}=\left(-\frac{d l_{i}}{d \alpha_{i}} \sin \left(\gamma_{i}+\frac{\alpha_{i}}{2}\right)-\frac{l_{i}}{2} \cos \left(\gamma_{i}+\frac{\alpha_{i}}{2}\right)\right), y_{\gamma i}=$ $-l_{i} \sin \left(\gamma_{i}+\frac{\alpha_{i}}{2}\right), y_{\alpha i}=\left(\frac{d l_{i}}{d \alpha_{i}} \cos \left(\gamma_{i}+\frac{\alpha_{i}}{2}\right)-\frac{l_{i}}{2} \sin \left(\gamma_{i}+\frac{\alpha_{i}}{2}\right)\right)$.

Thus :

$$
\mathbf{R}_{i+1}=\mathbf{R}_{i}+\left[\left[\begin{array}{c}
x_{\gamma i} \\
y_{\gamma i} \\
0 \\
0
\end{array}\right] \times \mathbf{1}_{1 \times(i-1)}\left|\begin{array}{c|c|c}
x_{\alpha i} & 0 \\
y_{\alpha i} & 0 & \mathbf{0}_{4 \times(N-i)} \\
1 & 0 \\
-1 & 1
\end{array}\right|\right.
$$

The inertia matrix $\mathbf{M}$ of the whole manipulator can then be expressed as:

$$
\mathbf{M}=\sum_{i=1}^{N} \mathbf{R}_{i}^{\top} \mathbf{M}_{i} \mathbf{R}_{i}
$$

For more simplicity in this study, the Coriolis effects are neglected in the control law and need not be calculated (see section 4).

\subsection{Potential energy for a stack of $\mathrm{N}$ mechanisms}

The potential energy of one mechanism is composed of the potential energy due to the mass of the different bars, and the potential energy due to the springs:

$$
\begin{aligned}
V_{g_{i}} & =g\left(m_{2 i} y_{2 i}+m_{3 i} y_{3 i}+m_{4 i} y_{4 i}\right) \\
V_{s p_{i}} & =\frac{1}{2} k_{l i}\left(l_{l i}-l_{0}\right)^{2}+\frac{1}{2} k_{r i}\left(l_{r i}-l_{0}\right)^{2}
\end{aligned}
$$

where $l_{0}$ is the free length of spring. The gravity and spring contribution to the equation of motion $\mathbf{G}$ can be written as :

$$
\mathbf{G}(\alpha)=\left[\frac{\partial V}{\partial \alpha_{1}} \ldots \frac{\partial V}{\partial \alpha_{i}} \ldots \frac{\partial V}{\partial \alpha_{N}}\right]^{\top}
$$

Since the spring lengths depend only on the internal geometry of the $i^{t h}$ mechanism (i.e $\alpha_{i}$ ), each component of $\mathbf{G}$ is computed as follows :

$$
G_{i}=\frac{\partial V}{\partial \alpha_{i}}=\frac{\partial}{\partial \alpha_{i}}\left(\sum_{k=i}^{N} V_{g_{k}}\right)+\frac{\partial V_{s p_{i}}}{\partial \alpha_{i}}
$$

\subsection{Generalized forces}

In this study, each mechanism is assumed fully actuated in an antagonist way, namely, a pair of tendons applies two positive forces $f_{l i}$ and $f_{r i}$ in parallel to the left and right spring, respectively. The generalized forces vector $\mathbf{Q}$ defined in (3) is the torque associated to the generalized coordinate $\boldsymbol{\alpha}$ :

$$
\mathbf{Q}=\mathbf{Z}_{l} \mathbf{f}_{l}+\mathbf{Z}_{r} \mathbf{f}_{r}
$$

with $\mathbf{f}_{l}=\left[f_{l 1}, \ldots, f_{l N}\right]^{\top}, \mathbf{f}_{r}=\left[f_{r 1}, \ldots, f_{r N}\right]^{\top}, \mathbf{Z}_{l}$ and $\mathbf{Z}_{r}$ are two diagonal matrices whose entries are respectively $-\frac{d l_{l i}}{d \alpha_{i}}$ and $-\frac{d l_{r i}}{d \alpha_{i}}, i=1, . . N$. 


\section{Control}

Each mechanism $i$ has a desired trajectory $\alpha_{i}^{d}$. Let $\boldsymbol{\alpha}^{d}$ be the vector of all those trajectories, and $\boldsymbol{\alpha}$ the vector of the measured mechanism orientations. The control law is built from equation (4). Low velocities are assumed and the Coriolis effects are thus neglected: $\mathbf{C}=\mathbf{0}_{N \times N}$. Thus, the desired torque $\mathbf{Q}_{d}$ is:

$$
\mathbf{Q}_{d}=\mathbf{M}(\boldsymbol{\alpha})\left(\ddot{\boldsymbol{\alpha}}^{d}+k_{d} \mathbf{I}_{N}\left(\dot{\boldsymbol{\alpha}}^{d}-\dot{\boldsymbol{\alpha}}\right)+k_{p} \mathbf{I}_{N}\left(\boldsymbol{\alpha}^{d}-\boldsymbol{\alpha}\right)\right)+\mathbf{G}(\boldsymbol{\alpha})
$$

where $k_{d}$ and $k_{p}$ are the gains of the control law. The required forces $\mathbf{f}_{l}$ and $\mathbf{f}_{r}$ must satisfy:

$$
\mathbf{Q}_{d}=\mathbf{Z}_{l}(\boldsymbol{\alpha}) \mathbf{f}_{l}+\mathbf{Z}_{r}(\boldsymbol{\alpha}) \mathbf{f}_{r}
$$

The solution that minimizes the norm of the sum of the forces $\left\|\mathbf{f}_{l}+\mathbf{f}_{r}\right\|$ is chosen i.e. since forces must remain positive, for each mechanism one force is zero and the other one produces the motion.

This dynamic control law is compared to a PD control law including G. For this control law, the desired torque is :

$$
\mathbf{Q}_{l d}=\mathbf{M}_{\mathbf{m}}\left(k_{d} \mathbf{I}_{N}\left(\dot{\boldsymbol{\alpha}}^{d}-\dot{\boldsymbol{\alpha}}\right)+k_{p} \mathbf{I}_{N}\left(\boldsymbol{\alpha}^{d}-\boldsymbol{\alpha}\right)\right)+\mathbf{G}(\boldsymbol{\alpha})
$$

where $\mathbf{M}_{\mathbf{m}}$ is a constant diagonal matrix built with the maximum of the diagonal elements of $\mathbf{M}$ for angular positions between $-\frac{\pi}{2}$ and $\frac{\pi}{2}$. Thus, the lower the mechanism in the stack, the bigger the gains of the PD control law.

\section{Simulation Results}

\subsection{Neck parameters}

The manipulator studied is made of $N=9$ identical mechanisms with $L=0.15 \mathrm{~m}$ and $b=0.1 \mathrm{~m}$. The springs free length is defined as $l_{0}=L-b$ (which is the smallest length of the springs reached in the flat configurations $\alpha_{i}= \pm \pi$ ). All bars are cylinders of diameter $d=0.01 \mathrm{~m}$ and made of ABS with a volumic mass $1050 \mathrm{~kg} / \mathrm{m}^{3}$. Since most birds have an S-shape rest configuration of their neck, such a rest configuration is chosen for our manipulator. It is defined by

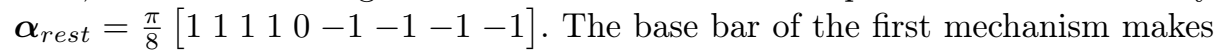
an angle $\gamma_{1}=-\frac{\pi}{4}$ with respect to the horizontal axis. Since the rest configuration is a stable equilibrium of the manipulator with zero-actuation forces, the two conditions below must be satisfied for each mechanism [12]:

$$
\left\{\begin{array}{l}
\mathbf{G}_{i}\left(\boldsymbol{\alpha}_{\text {rest }}\right)=0 \\
\frac{\partial \mathbf{G}_{i}}{\partial \alpha_{i}}\left(\boldsymbol{\alpha}_{\text {rest }}\right) \geq K_{\text {min }}
\end{array}\right.
$$

Here, we choose $K_{\min }=1 \mathrm{Nm} / \mathrm{rad}$. The $2 \mathrm{~N}=18$ equations in (22) are used to determine the 18 spring stiffnesses of the manipulator. Figure 2 shows the rest configuration and the spring stiffnesses obtained. Note that due to the effect of 

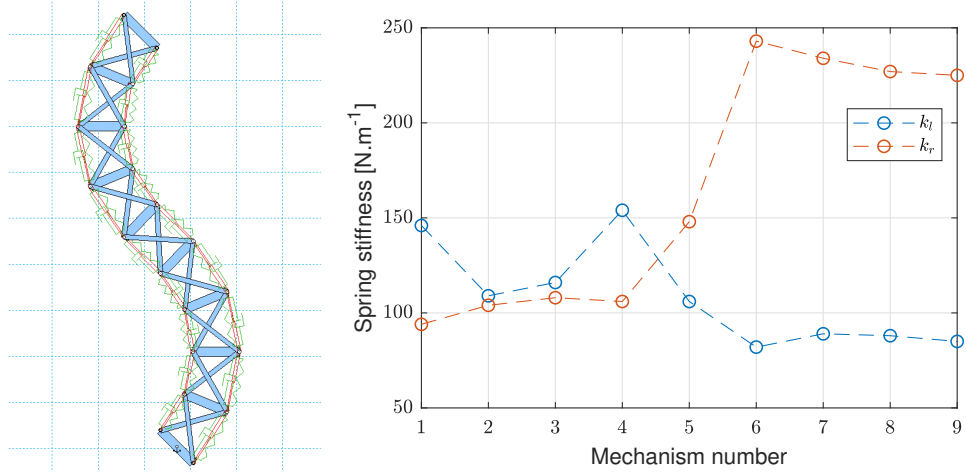

Fig. 2: Position at rest of the mechanism and spring stiffnesses

weight, we may obtain different spring stiffnesses for mechanisms with the same rest orientation.

The minimal and maximal bounds of the actuation forces are defined as $f_{\min }=0 \mathrm{~N}$ (the tendons can only pull) and $f_{\max }=100 \mathrm{~N}$, respectively.

\subsection{Control results}

Simulations are performed with Matlab and Working Model 2D ${ }^{\complement}$. Starting from its S-shape rest configuration, the manipulator is moved toward a straight configuration in five seconds where it is maintained straight during one second, and then returns to its rest configuration in five seconds. A skew sine displacement is defined between each desired configuration. It is worth noting that in the rest configuration, mechanism 5 has its equilibrium at $\alpha_{5 \text { rest }}=0$, thus it does not need to move during the whole trajectory. The desired trajectories are shown in black in Figure 3.

Figure 3 shows the positions of the mechanisms during the simulations. The blue curve, which corresponds to the PD control law, is close to the reference curve, however oscillations appear. Here, the gains are $k_{p}=38$ and $k_{d}=0.07$. The main problem of the PD control law is that the gains cannot be too high due to the oscillations, otherwise the manipulator becomes unstable.

Nevertheless the dynamic control law works well with higher gains. The red curve shows the configurations obtained with the dynamic control law with $k_{p}=$ 625 and $k_{d}=50$. In this case, the error is very low, except for the last mechanism, which has a small delay with respect to the desired trajectory. This is because the centrifugal and coriolis effects are not compensated whereas they becomes predominant for this module. Here again, no oscillations appear (see a video at http://perso.eleves.ens-rennes.fr/people/benjamin.fasquelle/english/phd.html).

The forces applied with the dynamic control law on each mechanism are shown in Figure 4. Here, the bar masses and inertia as well as the velocities are quite law. Consequently, the generalized forces deriving from the potential energy $\mathbf{G}$ is the major part of the desired torque $\mathbf{Q}_{d}$. 

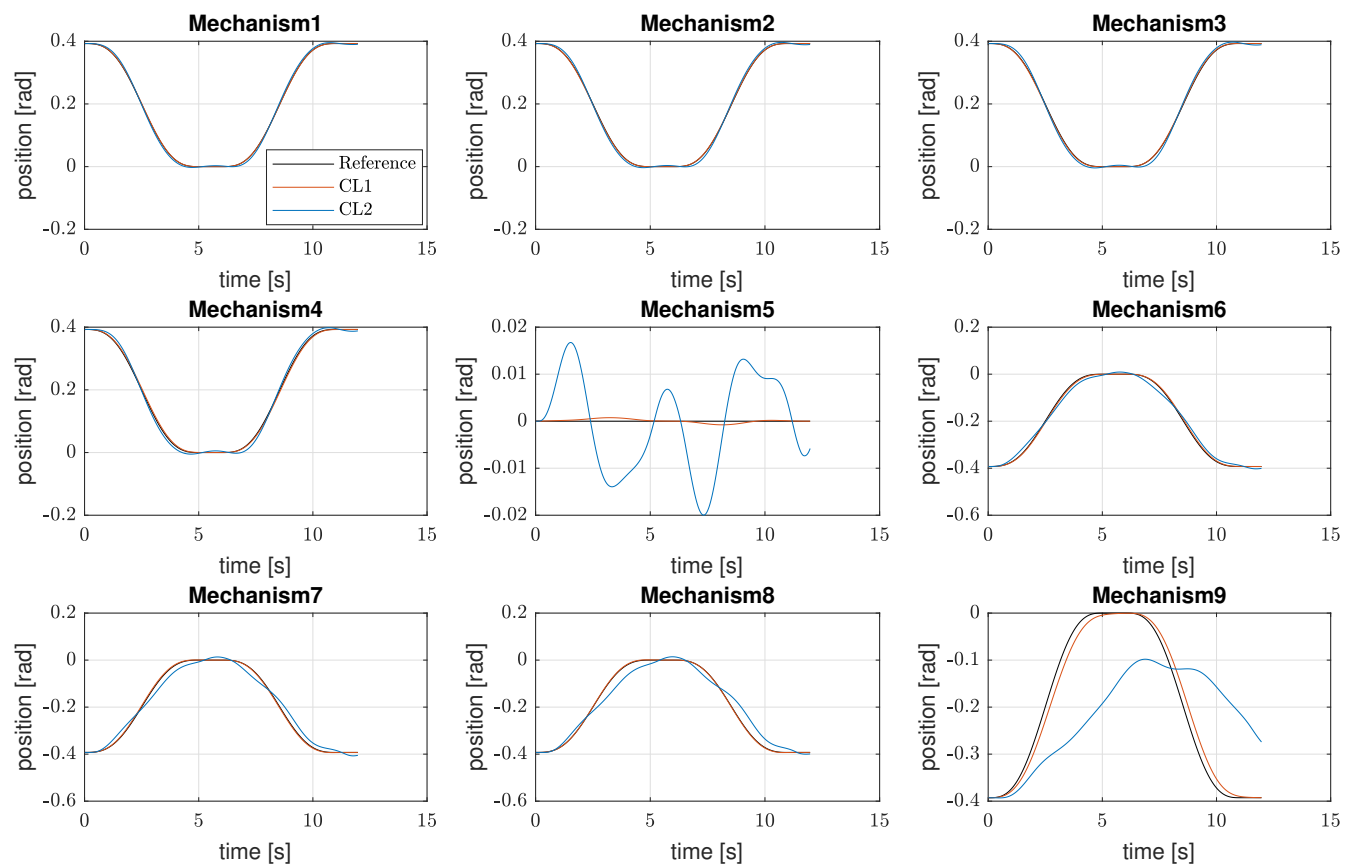

Fig. 3: Position during the simulations. The Reference curve (in black) corresponds to the desired trajectory, the CL1 (in red) one is the result of the dynamic control law and CL2 (in blue) is the result of the PD control law including $\mathbf{G}$.
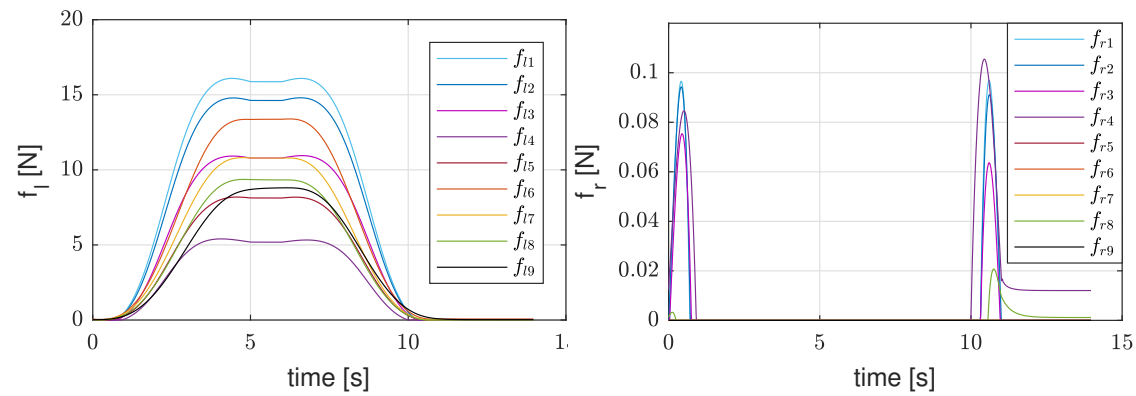

Fig. 4: Forces applied during the movement. 


\section{Conclusion}

This paper proposed an original, efficient method for the derivation of the inertia matrix of a tensegrity manipulator made of several X-shape mechanisms in series. A local inertia matrix was defined for each mechanism. This matrix was parametrized with the absolute pose of the reference frame $x_{i}, y_{i}, \gamma_{i}$ and one unique variable $\alpha_{i}$ defining the mechanism configuration. The complete inertia matrix was obtained in a simple way from the absolute coordinates of all mechanisms expressed as functions of the configuration variable $\boldsymbol{\alpha}$. Then, a dynamic control law was proposed for the tensegrity manipulator to track desired trajectories. It was shown that taking into account the inertia matrix, which accounts for the couplings between the individual mechanisms, allows for a reasonable damping of the shaking effects due to the inherent flexibilities, as well as for the use of sufficient gains for an acceptable system behavior.

Acknowledgement This work was conducted with the support of the French National Research Agency (AVINECK Project ANR-16-CE33-0025).

\section{References}

1. Motro, R. Tensegrity systems: the state of the art, Int. J. of Space Structures, 7 (2), pp 75-83, 1992

2. K. Snelson, 1965, Continuous Tension, Discontinuous Compression Structures, US Patent No. 3,169,611

3. R. B. Fuller, Tensile-integrity structures, United States Patent 3063521,1962

4. Skelton, R. and de Oliveira, M., Tensegrity Systems. Springer, 2009

5. M. Arsenault and C. M. Gosselin, Kinematic, static and dynamic analysis of a planar 2-dof tensegrity mechanism, Mech. and Mach. Theory, Vol. 41(9), 1072-1089, 2006

6. C. Crane et al., Kinematic analysis of a planar tensegrity mechanism with presstressed springs, in Advances in Robot Kinematics: analysis and design, pp 419-427, J. Lenarcic and P. Wenger (Eds), Springer (2008)

7. P. Wenger and D. Chablat, Kinetostatic Analysis and Solution Classification of a Planar Tensegrity Mechanism, proc. 7th. Int. Workshop on Comp. Kinematics, Springer, ISBN 978-3-319-60867-9, pp422-431, 2017.

8. Q. Boehler, M. Vedrines, S. Abdelaziz, P. Poignet, P. Renaud, Design and evaluation of a novel variable stiffness spherical joint with application to MR-compatible robot design. In Robotics and Automation (ICRA), 2016 IEEE International Conference on (pp. 661-667).

9. S. Levin, The tensegrity-truss as a model for spinal mechanics: biotensegrity, J. of Mechanics in Medicine and Biology, Vol. 2(3), 2002

10. G. Zweers, R. Bout, and J. Heidweiller, Perception and Motor Control in Birds: An Eco- logical Approach. Springer, 1994, ISBN: 978-3-642-75869-0.

11. Q. Boehler et al., Definition and computation of tensegrity mechanism workspace, ASME J. of Mechanisms and Robotics, Vol 7(4), 2015

12. A. Van Riesen et al, Dynamic Analysis and Control of an Antagonistically Actuated Tensegrity Mechanism, in Romansy 22 - Robot Design, Dynamics and Control, Spinger, ISBN: 978-3-319-78962-0, 2018

13. JB Aldrich and RE Skelton, Time-energy optimal control of hyper-actuated mechanical systems with geometric path constraints, in 44th IEEE Conference on Decision and Control, pp 8246-8253, 2005 
14. S. Chen and M. Arsenault, Analytical Computation of the Actuator and Cartesian Workspace Boundaries for a Planar 2-Degree-of-Freedom Translational Tensegrity Mechanism, Journal of Mech. and Rob., Vol. 4, 2012

15. D. L Bakker et al., Design of an environmentally interactive continuum manipulator, Proc.14th IFToMM World Congress in Mechanisms and Machine Science, Taipei, Taiwan, 2015

16. A. Van Riesen et al, Optimal Design of Tensegrity Mechanisms Used in a Bird Neck Model, in EuCoMeS2018: Proceedings of the 7th European Conference on Mechanism Science, Springer, ISBN: 978-3-319-98019-95

17. M. Furet et al., Kinematic analysis of planar tensegrity 2-X manipulators, Proc. 16th International Symposium on Advances in Robot Kinematics, Bologna, Italia, 2018

18. M. Furet et al., Workspace and cuspidality analysis of a 2-X planar manipulator, Proc. 4th IFToMM Symposium on Mechanism Design for Robotics, Udine, Italia, 2018

19. W. Khalil and E. Dombre, Modeling, identification and control of robots. HPS, 2002 